\title{
Hierarchical Models for Rescoring Graphs vs. Full Integration
}

\author{
Raquel Justo* and M. Inés Torres \\ Dpto. Electricidad y Electrónica, Universidad del País Vasco (UPV/EHU), Spain
}

\begin{abstract}
In this work, we explore the integration of hierarchical Language Models (HLMs) in different modules of a Spoken Dialog System. First of all, HLMs are integrated into the Automatic Speech Recognition system. In order to carry out this integration, within the recognition process, finite-state machines were considered. This approach was compared to a two step decoding process in which HLMs are used to rescore a graph. Then, HLMs were also used for Language Understanding (LU) purposes. Two architectures were compared theoretically and empirically in both ASR and LU modules.
\end{abstract}

Keywords: finite-state machines, language models, automatic speech recognition, language understanding.

\section{Introduction}

In a classical pattern recognition problem the maximization of posterior probability $P(\bar{w} \mid \bar{x})$ allows to get the most likely sequence of symbols $\bar{w}$, that matches a given sequence of input observations $\bar{x}$, according to eq (1).

$$
\hat{\bar{w}}=\arg \max _{\bar{w}} P(\bar{w} \mid \bar{x})=\arg \max _{\bar{w}} P(\bar{w}) P(\bar{x} \mid \bar{w})
$$

Automatic Speech Recognition (ASR) is a classical pattern recognition problem in which the term $P(\bar{w})$ corresponds to the prior probability of a word sequence and it is commonly estimated by a Language Model (LM), while $P(\bar{x} \mid \bar{w})$ is estimated by an Acoustic Model (AM), typically a Hidden Markov Model.

If we focus on $P(\bar{w})$, word n-gram LMs are the most widely used approach in ASR systems. However, a large amount of training material, that is not always available, is needed to get a robust estimations of the parameters of such models. Therefore, different alternatives like Hierarchical Language Models (HLMs) based on classes of phrases could be adopted. This approach has been successfully employed in ASR systems for restricted domain applications [6]. However, the integration of this kind of complex models into the ASR system is an important issue that has to be solved. Usually, decoders can deal with standard word-based LMs, but when complex LMs need to be used two possible alternatives can be considered: 1) a decoupled architecure consisting of a two-step

\footnotetext{
* This work has been partially supported by the Gov. of the Basque Country under
} grant IT685-13 and by the Spanish CICYT under grant TIN2011-28169-C05-04.

J. Ruiz-Shulcloper and G. Sanniti di Baja (Eds.): CIARP 2013, Part I, LNCS 8258, pp. 496-503, 2013. (C) Springer-Verlag Berlin Heidelberg 2013 
process with a word graph rescoring 92 or 2) an integrated architecture where the decoder has to be modified to integrate the LM into the recognition process.

An ASR system employs different knowledge sources to carry out the recognition process such as acoustic, phonetic, lexical and syntactic knowledge. Each of this sources adds complexity to the decoding algorithm. AT\&T laboratories presented an approach that simplifies the integration of different knowledge sources into the ASR system by using Stochastic Finite State Transducers (SFST) 831. Newer architectures have also been recently provided for large vocabulary ASR [10. The integrated architecture employed in this work takes advantage of the same idea, that is, the composition of finite-state machines, which model each knowledge source, to build a dynamic search network. This architecture was previously described in [7] and it is compared to a decoupled architecture.

On the other hand, HLMs can also be used to deal with a Language Understanding $(\mathrm{LU})$ procedure that is devoted to extracting semantic information from a text sentence.

The contribution of this paper is twofold: first, it provides a full theoretical description of the two architectures, i.e. fully integrated HLMs vs. HLMs for graphs rescoring. Then, these approaches are theoretically and empirically evaluated for both ASR and LU purposes in a Spanish Spoken Dialogue task. The paper is organized as follows: in Section 2 the employed LMs are briefly described. Then, in Section 3 a full theoretical description of the two architectures is given for ASR and LU tasks; and a theoretical comparison between them is given. Finally, Section 4 and Section 5 summarizes the obtained results and the extracted conclusions.

\section{HLMs Based on Classes of Phrases}

In this section we present the different LMs that were used in this work. All of them were defined as Stochastic Finite State models. Specifically, we used $k$-Testable in the Strict Sense ( $k$-TSS) LMs, which are a subclass of regular languages and have been successfully proposed for natural language processing applications [1]. They have been considered as a syntactic approach of the ngram LMs under certain conditions. First of all a classical word $k$-TSS LM was considered $\left(M_{w}\right)$, where the probability of a sequence of $N$ words $(\bar{w})$, is obtained considering the history of previous $k_{w}-1$ words as shown in eq. (2).

$$
P(\bar{w}) \simeq P_{M_{w}}(\bar{w})=\prod_{i=1}^{N} P\left(w_{i} \mid w_{i-k_{w}+1}^{i-1}\right)
$$

Then two different approaches for HLMs $\left(M_{s w}, M_{s l}\right)$ were considered [6]. In the first approach, $M_{s w}$, a set of classes made up of phrases constituted by not linked words is used. In this way, the probability of a word sequence $(\bar{w})$ can be computed by means of eq. (3), where the segmentation (s) and classification $(\bar{c})$ of a word sequence are considered as hidden variables:

$$
P(\bar{w})=\sum_{\forall \bar{c} \in \Sigma_{c}^{*}} \sum_{\forall s \in \mathcal{S}(\bar{w})} P(\bar{w}, \bar{c}, s)=\sum_{\forall \bar{c} \in \Sigma_{c}^{*}} \sum_{\forall s \in \mathcal{S}(\bar{w})} P(\bar{w} \mid s, \bar{c}) P(s \mid \bar{c}) P(\bar{c})
$$


being $\Sigma_{c}^{*}$ the set of all possible $\bar{c}$ given an a-priori defined $\Sigma_{c}=\left\{c_{i}\right\}$ alphabet of classes and being $S(\bar{w})$ the set of all possible segmentations of a given $\bar{w}$.

Then, assuming the following approaches: a $k$-TSS model to estimate the term $P(\bar{c})\left(k_{c}-1\right.$ stands for the considered history of classes $), P(s \mid \bar{c}) \simeq \alpha$ and $P(\bar{w} \mid s, \bar{c})$ estimated with zero order models $\left(k_{c w}-1\right.$ stands for the maximum length of the word history considered in each class), eq. (3) is rewritten as follows:

$$
P(\bar{w}) \simeq \alpha \sum_{\forall \bar{c} \in \Sigma_{c}^{*}} \sum_{\forall s \in \mathcal{S}(\bar{w})} \prod_{i=1}^{T}\left[\left[\prod_{j=a_{i-1}+1}^{a_{i}} P\left(w_{j} \mid w_{j-k_{c w}+1}^{j-1}, c_{i}\right)\right] P\left(c_{i} \mid c_{i-k_{c}+1}^{i-1}\right)\right]
$$

According to this, $N_{c}+1\left(N_{c}\right.$ is the size of the set of classes) SFSA are needed to represent the $M_{s w}$ model: one for each class considering the relations among words inside the classes, $P\left(w_{j} \mid w_{j-k_{c w}+1}^{j-1}, c_{i}\right)$, and an additional one that takes into account the relations among classes, $P\left(c_{i} \mid c_{i-k_{c}+1}^{i-1}\right)$.

In the second approach, $M_{s l}$, classes are made up of phrases constituted by linked words, $\bar{l}$. Thus, the probability of $\bar{w}$ is given now by eq. (5)

$$
P(\bar{w})=\sum_{\forall \bar{c} \in \Sigma_{c}^{*} \forall \bar{l} \in \Sigma_{l}^{*}} P(\bar{w}, \bar{c}, \bar{l})=\sum_{\forall \bar{c} \in \Sigma_{c}^{*} \forall} \sum_{\bar{l} \in \Sigma_{l}^{*}} P(\bar{w} \mid \bar{l}, \bar{c}) P(\bar{l} \mid \bar{c}) P(\bar{c})
$$

where $\Sigma_{c}^{*}$ is the set comprising all possible class sequences for the given $\Sigma_{c}$ alphabet of classes and $\Sigma_{l}^{*}$ is the set of all possible sequences of $l_{i}$ phrases. Assuming that $P(\bar{c})$ is estimated using a $k$-TSS model, that $P(\bar{l} \mid \bar{c})$ is estimated using zero-order models and finally, $P(\bar{w} \mid \bar{l}, \bar{c})$ is equal to 1 when $\bar{l}$ and $\bar{c}$ are compatible with $\bar{w}$ and 0 otherwise, the eq. (5) can be rewritten as eq. (6) ).

$$
P(\bar{w}) \simeq \sum_{\forall \bar{c} \in \mathcal{C}} \sum_{\forall \bar{l} \in \mathcal{L}_{\bar{c}}(\bar{w})} \prod_{i=1}^{T}\left[P\left(l_{i} \mid c_{i}\right) P\left(c_{i} \mid c_{i-k_{c}+1}^{i-1}\right)\right]
$$

$N_{c}+1$ SFSA are needed again to represent the $M_{s l}$ model: one that takes into account the relations among classes, $P\left(c_{i} \mid c_{i-k_{c}+1}^{i-1}\right)$, and $N_{c}$ additional SFSA that, in this case, stands for 1-TSS models, $P\left(l_{i} \mid c_{i}\right)$.

\section{$3 \quad$ HLMs into ASR and LU Systems}

When considering the ASR system, we want to carry out the recognition process by using the HLMs $\left(M_{s w}\right.$ and $\left.M_{s l}\right)$ defined above through two different architectures. First of all, an integrated architecture was considered. In this case, we took advantage of the use of $k$-TSS LMs. These models can be represented by SFSA, which can be easily composed with each other and with other automata. When considering the integration of complex HLMs there is not only one automaton associated to each LM but $N_{c}+1$ different SFSA are needed, as described in Section 2. Thus, for doing the integration of the different SFSA a dynamic composition was carried out, in the same way the different models involved in the recognition process (LM, AM and lexical models) are integrated into the search network for a classical word $k$-TSS LM. 


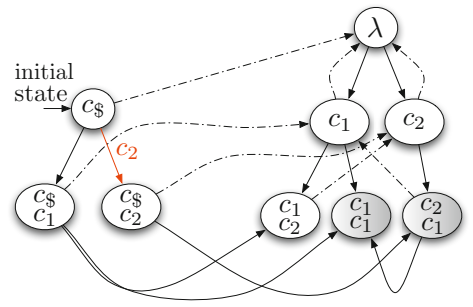

Fig. 1. SFSA that considers the relations among classes (shaded states correspond to final states)

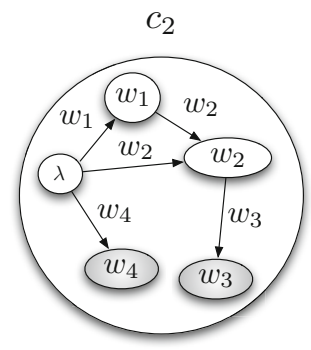

Fig. 2. Specific SFSA for a $c_{2}$ class (final states are shaded)

Let us show an example to illustrate this method for the $M_{s w}$ model. Being $\Sigma_{c}=\left\{c_{1}, c_{2}\right\}$ a two-class vocabulary made up of phrases, where $c_{1}=\left\{w_{1}, w_{1} w_{1}\right\}$ and $c_{2}=\left\{w_{2} w_{3}, w_{1} w_{2} w_{3}, w_{4}\right\}$, Fig 1 and Fig 2 represent the automata that take into account the relations among classes, and the specific automaton associated to class c2, respectively. Then, when the search network is built dynamically and the transition probabilities between words are needed (red arrows in Fig. 3) not only one but the two SFSA associated to the LM have to be consulted.

In this case, the probability of a word sequence is given by eq. (3) and the most likely word sequence is estimated according to the Viterbi algorithm:

$$
\hat{\bar{w}} \simeq \arg \max _{\forall \bar{w} \in \Sigma_{w}^{*}}\left[\max _{\forall \bar{c} \in \Sigma_{c}^{*} \forall s \in S_{c}(\bar{w})} \max _{\bar{n}} P(\bar{x}, \bar{n} \mid \bar{w}, \bar{c}, s) P(\bar{w} \mid \bar{c}, s) P(s \mid \bar{c}) P(\bar{c})\right]
$$

where $\bar{n}$ is a path of the search network that is associated to a specific classification $\bar{c}$, to a specific segmentation $s$ and to a specific word sequence $\bar{w}$. Let us point out that in the decoding process all the probabilities associated to the $M_{s w}$ model, as well as to lexical models and AMs, are involved. When considering $M_{s l}$ model, a similar process is carried out, but in this case the SFSA associated to each class is a 1-TSS model and the Viterbi algorithm leads to the following $\hat{w}$ :

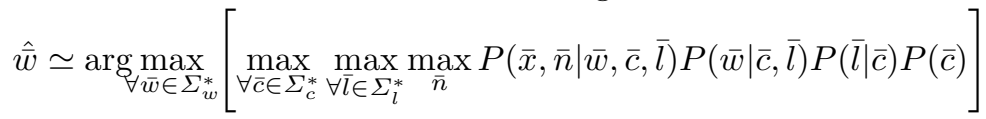

On the other hand, a decoupled architecture is considered when the recognition process is carried out by means of a two-pass decoder. First of all, we employ an standard decoder into which a classical word $k$-TSS LM $\left(M_{w}\right)$ is integrated. However, the decoder's output is not a word sequence $(\hat{\bar{w}})$, but a word-graph [5]. This word-graph produces a set of hypotheses denoted by $\Gamma_{G}(\bar{x})$, where $\Gamma_{G}(\bar{x}) \subset \Sigma_{w}^{*}$, being $\Sigma_{w}$ the alphabet of words. Once the word-graph has been obtained, we can now extract the $\mathcal{N}$-best list, $\mathcal{L}_{\mathcal{N}}(\bar{x})=\left\{\hat{\overline{w_{1}}}, \hat{\bar{w}_{2}}, \ldots, \hat{\overline{w_{\mathcal{N}}}}\right\}$, with its corresponding scores $\mathcal{P}_{\mathcal{N}}(\bar{x})=\left\{P\left(\hat{\overline{w_{1}}} \mid \bar{x}\right), P\left(\hat{\overline{w_{2}}} \mid \bar{x}\right), \ldots, P\left(\hat{\overline{w_{\mathcal{N}}}} \mid \bar{x}\right)\right\}$ where $\hat{\bar{w}}_{i} \in \mathcal{L}_{\mathcal{N}}(\bar{x})$, as shown in eq. (9) and (10).

$$
\begin{gathered}
\hat{\bar{w}}_{i} \simeq \underset{\forall}{\arg \max } \operatorname{mi\mathcal {L}_{i-1}^{c}(\overline {x})} P(\bar{x} \mid \bar{w}) P_{M_{w}}(\bar{w}) \simeq \underset{\forall \underset{w \in \mathcal{L}_{i-1}^{c}}{\arg \max } \min }{ }\left[\max _{\bar{n}} P(\bar{x}, \bar{n} \mid \bar{w}) P_{M_{w}}(\bar{w})\right] \\
P\left(\hat{\bar{w}}_{i} \mid \bar{x}\right) \simeq P\left(\bar{x} \mid \hat{\bar{w}}_{i}\right) P_{M_{w}}\left(\hat{\bar{w}}_{i}\right) \simeq \max _{\forall \bar{w} \in \mathcal{L}_{i-1}^{c}(\bar{x})}\left[\max _{\bar{n}} P(\bar{x}, \bar{n} \mid \bar{w}) P_{M_{w}}(\bar{w})\right]
\end{gathered}
$$




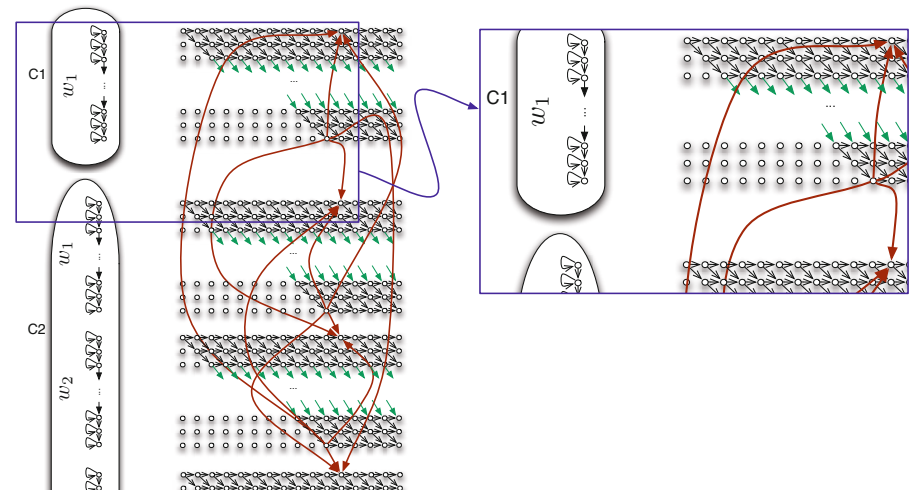

Fig. 3. Dynamic search network for $M_{s w}$ model

being $\mathcal{L}_{i-1}^{c}(\bar{x})=\Gamma_{G}(\bar{x})-\mathcal{L}_{i-1}(\bar{x})$; that is, the $i$-th best word sequence would be chosen among those hypotheses that are in $\Gamma_{G}(\bar{x})$ but without considering the ones that are already in the $(i-1)$-best list $\left(\mathcal{L}_{i-1}(\bar{x})\right)$.

Then, the HLM of choice $\left(M_{s w}\right.$ or $\left.M_{s l}\right)$ is employed to produce a new score for the obtained hypotheses (see eq. (3) ). A linear combination of this score and the one given in eq. (10) yields a final score, which is used to reorder the list again. Thus, we finally obtain a new best hypothesis (the system's output) when the HLM is used, as eq. (11) shows for the $M_{s w}$ model.

$$
\hat{\bar{w}} \simeq \underset{\bar{w}_{i} \in \mathcal{L}_{N}(\bar{x})}{\arg \max }\left[P\left(\hat{\bar{w}}_{i} \mid \bar{x}\right)+\alpha P_{M_{s w}}\left(\hat{\bar{w}}_{i}\right)\right]
$$

However, in the present work the sum over all $\bar{c}$ and $s$ is approaches by the maximum value. Therefore, eq. (11) can be rewritten as eq. (12) and in a similar way, $\hat{\bar{w}}$ would be estimated for $M_{s l}$ as eq. (13) shows:

$$
\begin{array}{r}
\hat{\bar{w}} \simeq \underset{\bar{w}_{i} \in \mathcal{L}_{N}(\bar{x})}{\arg \max }\left[P\left(\hat{\bar{w}}_{i} \mid \bar{x}\right)+\alpha \max _{\forall \bar{c} \in \Sigma_{c}^{*} \forall s \in \mathcal{S}_{c}\left(\hat{w}_{i}\right)} P\left(\hat{\bar{w}}_{i} \mid s, \bar{c}\right) P(s \mid \bar{c}) P(\bar{c})\right] \\
\hat{\bar{w}} \simeq \underset{\bar{w}_{i} \in \mathcal{L}_{N}(\bar{x})}{\arg \max }\left[P\left(\hat{\bar{w}}_{i} \mid \bar{x}\right)+\alpha \max _{\forall \bar{c} \in \Sigma_{c}^{*} \forall \bar{l} \in \Sigma_{l}^{*}} P\left(\hat{\bar{w}}_{i} \mid \bar{l}, \bar{c}\right) P(\bar{l} \mid \bar{c}) P(\bar{c})\right]
\end{array}
$$

If we compare eq. (12) and (13) with eq. (7) and (8), respectively, some differences can be observed. Here, the probabilities associated to $M_{w}$ LM are taken into account in the search network, providing $P\left(\hat{\bar{w}}_{i} \mid \bar{x}\right)$ values and then, the HLM is considered a posteriori. Although this architecture tries to simulate the integration of the model into the ASR system, the recognition process is not guided by the LM of choice. Turning to eq. (10) and (12), two maximization processes can be clearly differentiated. In the first one (eq. (10) ), the recognition process with $M_{w}$ is carried out and this LM together with lexical and acoustic models contribute to the local decisions until the final result is reached (an $\mathcal{N}$-best list $\left(\mathcal{L}_{N}(\bar{x})\right)$, in this case). Then, in the second maximization process (see eq. (12)) a hypothesis is chosen from among those that are in the list, according to the 
probability provided by the HLM. In this process the acoustic and lexical models do not take part in the decision-making process in any way. Furthermore, the result obtained with the decoupled architecture will always be limited by the best result that an $M_{w}$ model could provide using a word graph (oracle result), because it is selected from among those that are in $\mathcal{L}_{N}(\bar{x})$. On the other hand, this kind of integration enables us to use a simple standard decoder, while all the model's complexity is considered a posteriori, what could be very interesting in large-vocabulary ASR. When the integrated architecture is employed instead, a single maximization process is carried out, as can be seen in eq. (77), where lexical models and AMs along with the $M_{s w}$ model contribute to the local decision making until the resulting word sequence is obtained. The same happens with the $M_{s l}$ model (see eq. (8)).

In this work, we also propose to use hierarchical models within the LU module. When HLMs are integrated into the ASR system by using the integrated architecture, the Viterbi algorithm provides an estimation of the most-likely word sequence, as shown in eq. (77) and (8). However, these equations can be rewritten as eq. (14) and (15) respectively. Consequently, the word sequence, class sequence and segmentation associated to the most-likely sequence of states $\bar{n}$ can all be obtained simultaneously from the Viterbi algorithm.

$$
\begin{gathered}
{[\hat{\bar{w}}, \hat{\bar{c}}, \hat{s}] \simeq \underset{\forall \bar{w} \in \Sigma_{w}^{*}, \forall \bar{c} \in \Sigma_{c}^{*}, \forall s \in \mathcal{S}_{c}(\bar{w})}{\arg \max _{\bar{n}}}\left[\max _{\bar{n}} P(\bar{x}, \bar{n} \mid \bar{w}, \bar{c}, s) P(\bar{w} \mid \bar{c}, s) P(s \mid \bar{c}) P(\bar{c})\right]} \\
{[\hat{\bar{w}}, \hat{\bar{c}}, \hat{\bar{l}}] \simeq \underset{\forall \bar{w} \in \Sigma_{w}^{*}, \forall \bar{c} \in \Sigma_{c}^{*}, \forall \bar{l} \in \Sigma_{l}^{*}}{\arg \max _{\bar{n}}}\left[\max _{\bar{n}} P(\bar{x}, \bar{n} \mid \bar{w}, \bar{c}, \bar{l}) P(\bar{w} \mid \bar{c}, \bar{l}) P(s \mid \bar{c}) P(\bar{c})\right]}
\end{gathered}
$$

Thus, if our set of classes is obtained using a semantically motivated criterion, the semantic information associated to the word sequence will be retrieved during the recognition process. Therefore, when using the HLMs and the integrated ASR architecture, the speech recognition and understanding processes could be merged into a single step, thus speeding up the interventions of the dialogue system. Furthermore, the semantic, acoustic and language information contribute to the local decisions-making process throughout the search space until the output word sequence and class sequence are obtained. Thus, more accurate results could be attained regarding both words and classes.

In the same way, when HLMs are employed, the decoupled architecture can also be used to obtain the semantic information associated to a word sequence. Once the $\mathcal{N}$-best list $\mathcal{L}_{N}(\bar{x})$ has been obtained, all the possible $s$ and $\bar{c}$ associated to each hypothesis can be inferred. Using the hierarchical model of choice $\left(M_{s w}\right.$ or $\left.M_{s l}\right)$, a probability $P\left(\hat{\bar{w}}_{i}\right)$ is associated to each hypothesis according to eq. (3) and eq. (5) for $M_{s w}$ and $M_{s l}$ models respectively.

Then, a linear combination of this probability and $P\left(\hat{\bar{w}}_{i} \mid \bar{x}\right)$ is computed taking into account that in this work the sum over all different $s$ and $\bar{c}$ is approximated by the maximum. Finally, the class sequence associated to the best new score is provided in the output as eq. (16) and (17) show for the $M_{s w}$ and $M_{s l}$ models respectively.

$$
[\hat{\bar{w}}, \hat{\bar{c}}, \hat{s}] \simeq \underset{\forall \hat{\bar{w}}_{i} \in \mathcal{L}_{N}(\bar{x}), \forall \bar{c} \in \Sigma_{c}^{*}, \forall s \in \mathcal{S}_{c}\left(\hat{\bar{w}}_{i}\right)}{\arg \max }\left[P\left(\hat{\bar{w}}_{i} \mid \bar{x}\right)+\alpha P\left(\hat{\bar{w}}_{i} \mid s, \bar{c}\right) P(s \mid \bar{c}) P(\bar{c})\right]
$$




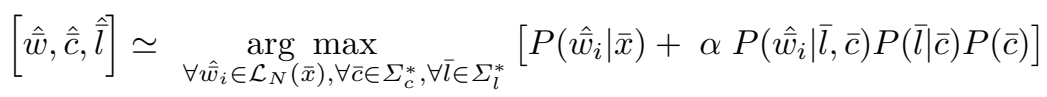

\section{Experimental Results}

In order to evaluate the approaches presented in this work a speech based conversational interface is considered. It consists of a virtual butler service that would be installed at home to control and provide information about electrical appliances. The system was developed within the framework of the GENIO project 4, which was partially supported by FAGOR. In this context a Spanish corpus (Domolab) was acquired with the specific features described in Table 1.

First of all, $M_{s w}$ and $M_{s l}$ models were evaluated when they were integrated into the ASR system. The two different architectures proposed in this work were considered here for comparison purposes and the results are shown in Table 2 From this table it can be concluded that hierarchical $M_{s w}$ and $M_{s l}$ models attain a better ASR system performance than the classical $M_{w}$ one, when using either integration method. However, the decoupled architecture provides slightly worst WER values than the integrated one for $M_{s w}$. Note that the lowest WER that can be achieved with the $M_{w}$ model through the word graph (oracle WER) is higher than the WER obtained with the integrated $M_{s w}$. Thus, a rescoring procedure cannot outdo this result.

We also evaluated HLMs within a LU task. Note that 40 manually-chosen, task-dependent semantic classes made up of phrases, specifically those employed in the LU module of the dialogue system, were used to build HLMs in both experiments. The evaluation was carried out in terms of the Category Error Rate (CER) and it is also presented in Table 2. CER is measured in the same way the WER is, but considering the class sequences provided by the system and the classified reference sentences. Table 2 shows that both architectures provide better CER values than the baseline $M_{w}$ model. Regarding this $M_{w}$ model, CER values were obtained by classifying both the reference sentences and the sentences obtained with the ASR system and an $M_{w}$ model. Consequently, it can be concluded that the extraction of the semantic information can benefit from the use of hierarchical models. Moreover, the percentage improvement associated

Table 1. Features of Domolab corpus

\begin{tabular}{|c|c|c|}
\hline & & Domolab \\
\hline \multicolumn{2}{|c|}{ Sentences } & 44,236 \\
\hline \multirow{3}{*}{ 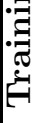 } & Diff. sent. & 43,962 \\
\hline & Words & 349,890 \\
\hline & Vocab. & 357 \\
\hline \multirow{3}{*}{$\vec{w}$} & Sentences & 1,617 \\
\hline & Words & $\overline{9,660}$ \\
\hline & Vocab. & 325 \\
\hline
\end{tabular}

Table 2. WER and CER results for $M_{w}, M_{s w}$ and $M_{s l}$ models using different architectures

\begin{tabular}{|c|c|c|c|c|c|}
\cline { 3 - 4 } \multicolumn{2}{c|}{} & \multicolumn{2}{|c|}{$M_{w}$} & \multirow{2}{*}{$M_{s w}$} & \multirow{2}{*}{$M_{s l}$} \\
\cline { 3 - 5 } \multicolumn{2}{c|}{} & 1-best & oracle & & \\
\hline $\begin{array}{c}\text { WER } \\
(\%)\end{array}$ & Int. Arch. & 6.04 & - & 5.14 & 5.4 \\
\hline Dec. Arch. & 6.04 & 5.23 & 5.34 & 5.35 \\
\hline $\begin{array}{c}\text { CER } \\
(\%)\end{array}$ & Int. Arch. & 8.68 & - & 7.18 & 7.06 \\
\cline { 2 - 4 } & Dec. Arch. & 8.68 & 5.93 & 7.12 & 6.98 \\
\hline
\end{tabular}


to the use of HLMs with regard to $M_{w}$ model, is greater when considering CER values than when considering WER values (e.g. $14.9 \%$ vs. $17.28 \%$ for $M_{s w}$ model and integrated architecture). Thus, the CER improvement is not only due to a better recognized sentence, but the semantic information involved in the HLMs is helping to provide a better CER result.

Regarding the two different architectures very similar performance values are achieved. However, when using integrated architecture all the processes (recognition and LU) can be carried out in only one step. Instead, decoupled architecture makes it possible to use HLMs to carry out the LU process over the outputs of different ASR systems.

\section{Concluding Remarks}

In this work the integration of complex HLMs into ASR systems was explored by making use of two different architectures, an integrated one and a decoupled one. The obtained results show that HLMs provide better ASR performance for both architectures. On the other hand, HLMs can also be used in a LU module. Different experiments were carried out in order to evaluate this task and the results show that this process can benefit from the use of hierarchical models.

\section{References}

1. Caseiro, D., Trancoso, I.: A specialized on-the-fly algorithm for lexicon and language model composition. IEEE TASLP 14(4), 1281-1291 (2006)

2. Chung, E., Jeon, H.B., Park, J.G., Lee, Y.K.: Lattice rescoring for speech recognition using large scale distributed language models. In: Proceedings of COLING, pp. 217-224 (2012)

3. Dolfing, H.J.G.A., Hetherington, I.L.: Incremental language models for speech recognition using finite-state transducers. In: Proceedings of IEEE ASRU, pp. 194197. Madonna di Campiglio, Italy (2001)

4. GENIO: Gestor Embebido Natural de Interfaz Oral. INTEK project. Industry Department. Basque Government (2006)

5. Justo, R., Pérez, A., Torres, M.I.: Impact of the approaches involved on word-graph derivation from the ASR system. In: Vitrià, J., Sanches, J.M., Hernández, M. (eds.) IbPRIA 2011. LNCS, vol. 6669, pp. 668-675. Springer, Heidelberg (2011)

6. Justo, R., Torres, M.I.: Phrase classes in two-level language models for asr. Pattern Analysis \& Applications 12(4), 427-437 (2009)

7. Justo, R., Torres, M.I.: Using finite state models for the integration of hierarchical LMS into ASR systems. In: Martínez-Trinidad, J.F., Carrasco-Ochoa, J.A., BenYoussef Brants, C., Hancock, E.R. (eds.) MCPR 2011. LNCS, vol. 6718, pp. 332340. Springer, Heidelberg (2011)

8. Pereira, F., Riley, M.D.: Speech recognition by composition of weighted finite automata. In: Finite-State Language Processing, pp. 431-453. MIT Press (1996)

9. Rastrow, A., Dredze, M., Khudanpur, S.: Fast syntactic analysis for statistical language modeling via substructure sharing and uptraining. In: Proceedings of ACL 2012, Stroudsburg, PA, USA, pp. 175-183 (2012)

10. Siniscalchi, S.M., Svendsen, T., Lee, C.H.: A bottom-up modular search approach to large vocabulary continuous speech recognition. IEEE TASLP 21(4), 786-797 (2013)

11. Torres, M.I., Casacuberta, F.: Stochastic k-tss bi-languages for machine translation. In: Proceedings of FSMNLP, pp. 98-106. ACL (2011) 